\title{
Correlation Between Protein Primary Structure and Soluble Expression Level of HSA dAb in Escherichia coli
}

Yankun Yang ${ }^{1,2}$, Guoqiang Liu',2, Meng Liu'², Zhonghu $\mathrm{Bai}^{2,3}$, Xiuxia Liu ${ }^{2,3}$, Xiaofeng $\mathrm{Dai}^{2,3 *}$ and Wenwen Guo ${ }^{3,4 *}$

${ }^{1}$ The Key Laboratory of Carbohydrate Chemistry and Biotechnology, School of Biotechnology, Jiangnan University, Ministry of Education, 1800 Lihu Avenue, 214122 Wuxi, PR China

${ }^{2}$ National Engineering Laboratory for Cereal Fermentation Technology, Jiangnan University, 1800 Lihu Avenue, 214122 Wuxi, PR China 3 Jiangsu Provincial Research Center for Bioactive Product Processing Technology, Jiangnan University, 1800 Lihu Avenue, 214122 Wuxi, PR China

${ }^{4}$ The Key Laboratory of Industrial Biotechnology, Ministry of Education, School of Biotechnology, Jiangnan University, 1800 Lihu Avenue, 214122 Wuxi, PR China

Received: July 29, 2017 Accepted: January 31, 2018

*Corresponding authors:

Phone/Fax: +8651085329306; E-mail:xiaofeng.dai@jiangnan.edu.cn (Dai), wendy_gww@foxmail.com (Guo)

ORCID IDs: 0000-0003-4815-6832 (Yang), 0000-0002-9754-587X (Liu, G), 0000-0002-8815-7429 (Liu,M), 0000-0003-3985-7434 (Bai), 0000-0003-1174-2897 (Liu, X), 0000-0002-0006-4042 (Dai), 0000-0002-7145-0303 (Guo)

Paper was presented at the 7th International Forum on Industrial Bioprocessing - IFIBiop 2017, May 21-24, 2017, Wuxi, PR China

\section{SUMMARY}

It is widely accepted that features such as pl, length, molecular mass and amino acid (AA) sequence have a significant influence on protein solubility. Here, we mainly focused on AA composition and explored those that most affected the soluble expression level of human serum albumin ( $\mathrm{HSA}$ ) domain antibody (dAb). The soluble expression and sequence of $65 \mathrm{dAb}$ variants were analysed using clustering and linear modelling. Certain AAs significantly affected the soluble expression level of $d A b$, with the specific $A A$ combinations being $(S, R, N, D, Q),(G, R, C, N, S)$ and $(R, S, G)$; these combinations respectively affected the $\mathrm{dAb}$ expression level in the broth supernatant, the level in the pellet lysate and total soluble $\mathrm{dAb}$. Among the $20 \mathrm{AAs}$, R displayed a negative influence on the soluble expression level, whereas $\mathrm{G}$ and $\mathrm{S}$ showed positive effects. A linear model was built to predict the soluble expression level from the sequence; this model had a prediction accuracy of $80 \%$. In summary, increasing the content of polar AAs, especially $G$ and $S$, and decreasing the content of $R$, was helpful to improve the soluble expression level of HSA dAb.

Key words: domain antibody (dAb), Escherichia coli, heterologous protein soluble expression, linear modelling, primary structure

\section{INTRODUCTION}

Given the outstanding advantages of Escherichia coli, including fast growth, inexpensive culturing, high-density cultivation, and simple genetic manipulation, it has been suggested that $E$. coli should be the first host tried for expression of any protein (1). However, most proteins from eukaryotes have low solubility when expressed in E. coli. For instance, over $80 \%$ of non-membrane proteins were unsuitable for structural studies and over $90 \%$ of potential pharmaceutical proteins were terminated at an early stage of clinical development because of their low solubility when expressed in E. coli (2). Several strategies have been used to increase protein production and solubility, for example altering expression system elements $(3,4)$ and optimizing culture conditions (5). These efforts are time-consuming, costly and usually difficult (6) because of a lack of understanding of the correlation between the effect of the expression system components and the characteristics of the expressed protein.

Interestingly, it has been found that primary structure features have a great impact on protein overexpression in $E$. coli $(7,8)$. Several prediction models have been established $(6,9)$, such as the Harrison prediction model (10), multiple linear regression (MLR) model (11), solubility index-based model (12), support vector machine-based model $(13,14)$, PROSO model (15), SOLpro model (16), cc SOL model (17) and PROSO II model (18). These bioinformatics models can significantly reduce trial and error procedures involved in optimization of expression systems to increase the soluble expression level of heterologous proteins. However, there has been limited application of these prediction models, partly because of the significant differences among the proteins chosen for building them and also because of the adoption of inconsistent culture conditions for expression of proteins $(6,8,9)$.

Domain antibodies (dAbs), which consist of only variable regions of heavy $\left(\mathrm{V}_{\mathrm{H}}\right)$ or light $\left(V_{L}\right)$ chains (19), have simple tertiary structures (Fig. 1;20,21), thus it is helpful to focus on 


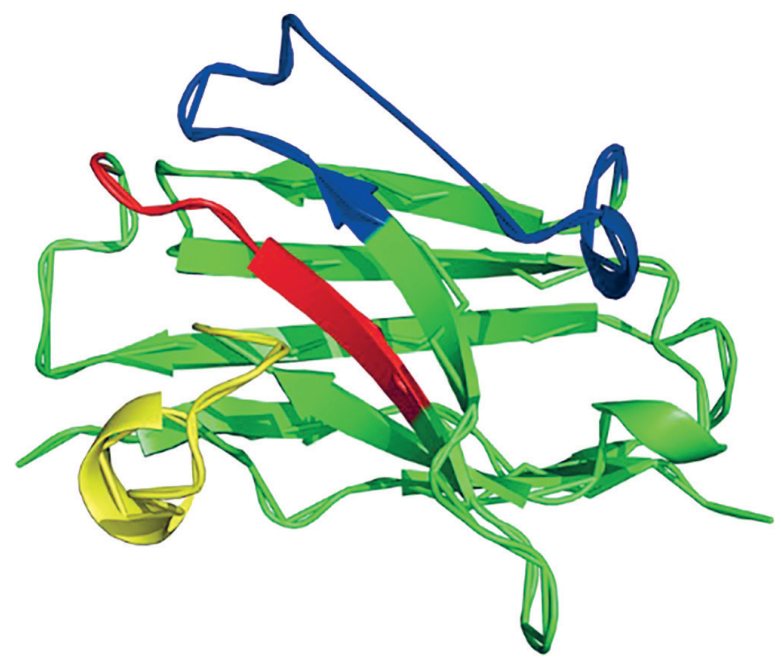

Fig. 1.3D structure of human serum albumin (HSA) domain antibody $(d A b)$ used in this study. 3D structure was obtained from SWISS-MODEL (20) and complementarity-determining regions (CDRs) were decorated in three different colours (red, yellow and blue) by PyMOL (21)

the features that influence $d A b$ expression level on primary structures. There are three hypervariable regions in dAbs, namely complementarity-determining regions (CDRs) I, II and III, where sequence variability is concentrated to determine the antigen-binding activity of an antibody (22). Small variations of amino acids (AAs) within a short region leading to clear variation in soluble expression level, ease of expression in E. coli (23), and a simple tertiary structure make dAbs an ideal model molecule to investigate the connections between primary structure features and the corresponding soluble protein expression levels.

In this study, a single expression system was used to express multiple human serum albumin (HSA) dAb variants with identical culture and detection conditions, to ensure that no other factors such as culture conditions affect the $d A b$ expression. Clustering and stepwise regression were used to explore the correlation between AA sequences and soluble expression levels of HSA dAbs, aiming at building a linear regression model to predict the soluble expression level of $\mathrm{HSA} d \mathrm{~d}$ based simply on its AA sequence. Such a model may act as a general guide for site-directed mutagenesis of HSA dAbs or other similar dAbs/Abs to improve the soluble expression levels, which benefits further studies such as interaction mechanism and structure research.

\section{MATERIALS AND METHODS}

\section{Random mutation of AAs in the CDRs of the original HSA $d A b$}

Five amino acids (AAs) were chosen in each complementarity-determining region (CDR) (there are three CDRs, so 15 AAs in total were chosen) to mutate randomly into other AAs, in this way we generated a mutation library consisting of about $10^{7}$ samples. These samples varied little in $\mathrm{pl}$ and molecular mass and had the same length, thus it was helpful to focus on the variables of AA composition. Then, $65 \mathrm{mu}-$ tated HSA dAbs excluding terminator mutants (AUA, CCU, CCC, AGA and AGG) or sequential repeat mutants were chosen randomly as experimental subjects and 10 were chosen as verification subjects. These mutated sequences are listed in Table 1.

\section{Production of recombinant $d A$ b expressing $E$. coli strains}

The $d A b$ fragments were cloned into vector pBY (an efficient expression vector constructed by a coworker in our lab) and introduced into E. coli strain BL21(DE3). The transformed cells were plated onto Luria-Bertani (LB) agar plates (Solarbio ${ }^{\circledR}$ Life Sciences, Beijing, PR China) and incubated at $37{ }^{\circ} \mathrm{C}$ overnight. After that, single colonies were selected and inoculated into $25 \mathrm{~mL}$ of LB medium (containing $15 \mu \mathrm{g}$ / $\mathrm{mL}$ of tetracycline (Shanghai Shenggong Co. Ltd., Shanghai, PR China) in $250-\mathrm{mL}$ flasks and incubated at $37^{\circ} \mathrm{C}$ for 7 $\mathrm{h}$ with shaking at $230 \mathrm{rpm}$. Stock solutions were prepared by mixing $500 \mu \mathrm{L}$ of culture with $500 \mu \mathrm{L}$ of $20 \%$ glycerol (Shanghai Hushi Laboratorial Equipment Co. Ltd., Shanghai, PR China) solution in $1.5-\mathrm{mL}$ tubes, and the cells were stored at $-80^{\circ} \mathrm{C}$.

\section{Cultivation of E. coli strains}

Cultivation can be divided into three phases: seed culture, growth and induction phase. Forty-eight square multititer plates (48-MTP; Thermo Fisher Scientific, Shanghai, PR China) were used to culture the 66 strains ( 65 mutated strains and a control strain) to achieve parallel fermentation. In the seed culture phase, $2 \mathrm{~mL}$ of LB medium containing $15 \mu \mathrm{g} / \mathrm{mL}$ of tetracycline were added into each well of the 48-MTP. After inoculation with $20 \mu \mathrm{L}$ of stock cell solution, 48-MTPs were incubated in a shaker at $230 \mathrm{rpm}$ and $30^{\circ} \mathrm{C}$ for $16 \mathrm{~h}$. In the growth phase, the seed solutions were transferred to fresh 48-MTPs containing $2 \mathrm{~mL}$ of Terrific Broth/Super Broth (TB/ $\mathrm{SB}$; Solarbio ${ }^{\circledR}$ Life Sciences) medium with $15 \mu \mathrm{g} / \mathrm{mL}$ of tetracycline and cultured under the same conditions as described above. The inoculum volume was calculated by the following equation, thus fixing the initial $A_{595 \mathrm{~nm}}$ at 0.05 :

$V($ inoculum $)=(0.05 \cdot V($ fermentation $) / A($ seed culture $)) / \mathrm{mL} \quad / 1 /$

where $V$ is the volume, 0.05 is the initial absorbance $(A)$ at 595 $\mathrm{nm}$ and $A$ is the absorbance of seed culture solution.

Seven hours after the second inoculation, isopropyl- $\beta$-D-thiogalactoside (IPTG; Solarbio ${ }^{\circledR}$ Life Sciences) was added to each well to a final concentration of $0.1 \mathrm{mM}$ and the culture temperature was lowered to $23^{\circ} \mathrm{C}$ simultaneously. The induction phase lasted for $16 \mathrm{~h}$. After centrifugation of the culture broth at $6000 \times g$ (centrifuge model Sorvall ST 16R; Thermo Fisher Scientific, Shanghai, PR China), the supernatants were collected, the cell pellets were resuspended in phosphate-buffered saline (PBS; Shanghai Hushi 
Table 1. Mutation results of 15 animo acids in complementarity-determining regions (CDRs) of human serum albumin (HSA) domain antibodies (dAb)

\begin{tabular}{|c|c|c|c|c|c|c|c|}
\hline No. & CDR1 & CDR2 & CDR3 & No. & CDR1 & CDR2 & CDR3 \\
\hline 1 & SQPHA & WLE-K & QFKHS & 34 & QYKDG & LLTHN & RNGAN \\
\hline 2 & KGNLR & CCSLR & PASTS & 35 & RTPQM & WNVNV & KGGVL \\
\hline 3 & RQYCP & AGVST & T-FMG & 36 & QQLTL & QSWTL & FCALL \\
\hline 4 & ----- & IAYSA & QFYWE & 37 & $-K N K P$ & RRASI & PVSGN \\
\hline 5 & ISNHW & ERVSN & QKFGV & 38 & LPKRL & GFLWI & NKLWQ \\
\hline 6 & YTPLY & FWR-Y & MHLML & 39 & DPREP & VMVKW & P-YDV \\
\hline 7 & PKFCL & SFEGG & KDNYL & 40 & NNNRR & PRYLF & NLHSA \\
\hline 8 & SRCVH & SPA-G & NNYHK & 41 & LDKNA & VLLIC & FGWPV \\
\hline 9 & RGPLS & WTTVL & DK-FT & 42 & DVCFK & LT-AS & WATSN \\
\hline 10 & SYIVP & RAVL- & NLGYL & 43 & WTLCS & VDTAR & FL-RS \\
\hline 11 & $-\mathrm{PRHL}$ & CGMTS & WGISP & 44 & ISKST & IPYCQ & NILQL \\
\hline 12 & TVPYR & ALTIG & -KSMS & 45 & KYHQS & RLLLE & KLTLL \\
\hline 13 & PSSIY & CCVDV & WRYEA & 46 & TIWKY & GFVLC & QINEK \\
\hline 14 & RLCPY & NSLGL & $\mathrm{SRCHY}$ & 47 & SVGAD & VSVAP & STR-N \\
\hline 15 & TP-VT & VSQ-Q & KTGPL & 48 & YDIGH & QRSRR & AADSD \\
\hline 16 & RWSFR & RTTQN & VNPMR & 49 & ----- & ----- & KLQCT \\
\hline 17 & SGLPT & FTWLI & ETPAL & 50 & GGLSL & GWLTT & IMT-K \\
\hline 18 & ----- & VNG-T & QFTGS & 51 & RANYN & RLGAA & HNMLQ \\
\hline 19 & YYLFS & EFIR- & SCALA & 52 & TAVT- & TA-LP & DEPMR \\
\hline 20 & -RPGL & ASALA & SAVRA & 53 & QL-F- & SWLAS & VDRAA \\
\hline 21 & QNRWL & -GLSS & $-\mathrm{K}-\mathrm{CP}$ & 54 & EASPR & VNVVP & GLNMR \\
\hline 22 & NTPFL & GNGLV & VNNNN & 55 & GA-VG & ----- & GSVCN \\
\hline 23 & FVITQ & MLRQT & -AYVA & 56 & SQSSQ & PFLFF & CYLPL \\
\hline 24 & AVGTW & DDARS & MAQLA & 57 & CRLTC & LRLQH & VNLQE \\
\hline 25 & AHNAE & PLSLP & SMSCF & 58 & NRNTG & GFLWI & NKLWQ \\
\hline 26 & SILTG & QNCWC & -RNHA & 59 & WCEPS & SAAQS & NSFFE \\
\hline 27 & VPHGG & FRRVN & RVSSK & 60 & ALGCC & FHDSR & SQNTV \\
\hline 28 & TIQQA & CDL-T & VCTGW & 61 & $-Y R H Q$ & YTFWT & YGCSK \\
\hline 29 & YTPPR & TG--N & SFWNP & 62 & CTKTL & ----- & VLAVM \\
\hline 30 & DIAGN & RV-HL & QRMKK & 63 & GTITQ & GTSTT & -TYLT \\
\hline 31 & TPESR & C-SES & DGQSD & 64 & SHYNQ & APVES & -VNGL \\
\hline 32 & ILFNL & ----- & SCMAS & 65 & NHAVK & -PIYL & KINTP \\
\hline 33 & LRSLE & D-TSV & MMDLW & Ori & HETMV & HIPPD & LPKRG \\
\hline V1 & SRKWC & DF-FT & RVLGW & V6 & PA-YP & AYVES & AAEKH \\
\hline V2 & SLRAD & QCKFL & RWHTA & V7 & SPHEE & CLT-Y & NNRPW \\
\hline V3 & SVEPS & LKMLG & IYQAT & V8 & KVDTR & RHGQL & CLHPT \\
\hline V4 & VTRSG & SGSDS & NIIST & V9 & ----- & ----- & Al-DN \\
\hline V5 & SHN-L & SRQWQ & VDATQ & V10 & YIPLF & GTIRA & TCWLH \\
\hline
\end{tabular}

- no alteration of amino acid at that position

Laboratorial Equipment Co. Ltd) and lysed using Precellys 24 (Bertin Technologies, Paris, France), and then supernatants were collected.

The whole process of cultivation was repeated six times; batches with small deviation of $\mathrm{dAb}$ production by control strain were chosen for further analysis, and in this way, parallel operations were guaranteed.

\section{Detection and quantification of soluble $d A b$ protein and total protein}

Two amounts of soluble expression of $d A b s$ were measured by direct ELISA, i.e. soluble dAbs in broth supernatant and in pellet lysate supernatant. Flat-bottomed 96-well plates (Thermo Fisher Scientific) were first coated with $50 \mu \mathrm{L}$ of supernatant. After blocking with $5 \%$ non-fat milk in PBS with 
Tween 20 (PBST; Shanghai Hushi Laboratorial Equipment Co. Ltd), the dAbs were detected using HRP-labelled protein A (Boster Biological Technology Co. Ltd., Beijing, PR China) with the substrate tetramethylbenzidine (Zhengzhou Biocell Biotechnology Co. Ltd., Zhengzhou, PR China). The reactions were stopped by the addition of $100 \mu \mathrm{L}$ of $2 \mathrm{M}$ sulfuric acid, and the absorbance was measured at $450 \mathrm{~nm} / 620 \mathrm{~nm}$ using an EZ Read 800 (Biochrom, Cambridge, UK). The amount of $\mathrm{dAb}$ was calculated from a standard curve made using reference sample. Total protein mass fraction was detected using a modified Bradford protein assay kit (Sangon Biotech Co. Ltd., Shanghai, PR China). To avoid the difference caused by different degrees of cell lysis, standardized amounts of dAbs in $\mu \mathrm{g}$ per $\mathrm{g}$ of total protein were calculated as follows and used in the data analysis (Table 2):

$$
w(\text { total protein })=m(\mathrm{dAb}) / m(\text { total protein })
$$

\section{Data analysis}

The software package $R$ (24) was used to analyze the contributions of factors such as AA composition, $\mathrm{dAb}$ charge and polarity on dAb soluble expression level. Factors with $\mathrm{p}<0.05$ were considered significant. Categories of AAs based on Vector $\mathrm{NTI}^{\circledR}(25)$ are listed in Table 3. Two levels of analysis were run, including dividing expression levels into high and low by Clustal Omega (26), and identifying the factors that had an effect on the expression level by $t$-test. A linear regression model was constructed, then factors that had a significant influence were removed in turn to identify the most significant ones based on Akaike information criterion (AIC) values (27). We used SWISS-MODEL (20) to get 3D structure, and PyMOL (21) to decorate CDRs in three different colours.

Table 2. Soluble expression data of 65 domain antibody (dAbs) variants using clustering and linear modelling

\begin{tabular}{|c|c|c|c|}
\hline No. & $\gamma($ soluble $d A b$ in supernatant $) /(n g / \mu L)$ & $\gamma($ soluble $\mathrm{dAb}$ in pellet)/(ng/ $\mu \mathrm{L})$ & $\gamma($ total soluble $d A b) /(n g / \mu L)$ \\
\hline 1 & $3.3 \pm 0.4$ & $4.3 \pm 0.7$ & $3.5 \pm 0.2$ \\
\hline 2 & $3.6 \pm 0.6$ & $5.0 \pm 0.3$ & $3.9 \pm 0.5$ \\
\hline 3 & $4.0 \pm 0.8$ & $4.2 \pm 0.2$ & $4.0 \pm 0.6$ \\
\hline 4 & $4.0 \pm 0.8$ & $4.3 \pm 0.4$ & $4.1 \pm 0.6$ \\
\hline 5 & $5.0 \pm 1.1$ & $3.8 \pm 0.2$ & $4.6 \pm 0.7$ \\
\hline 8 & $3.6 \pm 0.3$ & $2.3 \pm 0.3$ & $3.1 \pm 0.3$ \\
\hline 9 & $3.4 \pm 0.5$ & $4.3 \pm 1.0$ & $3.6 \pm 0.6$ \\
\hline 10 & $3.2 \pm 0.4$ & $4.5 \pm 0.8$ & $3.5 \pm 0.4$ \\
\hline 11 & $3.2 \pm 0.3$ & $3.1 \pm 0.9$ & $3.1 \pm 0.5$ \\
\hline 13 & $3.5 \pm 0.7$ & $4.5 \pm 1.1$ & $3.7 \pm 0.8$ \\
\hline 15 & $3.4 \pm 0.6$ & $2.5 \pm 0.5$ & $3.0 \pm 0.5$ \\
\hline 17 & $5.6 \pm 0.5$ & $4.2 \pm 1.1$ & $5.0 \pm 0.4$ \\
\hline 19 & $3.7 \pm 0.2$ & $4.0 \pm 1.0$ & $3.8 \pm 0.4$ \\
\hline 20 & $3.7 \pm 0.6$ & $3.1 \pm 0.6$ & $3.5 \pm 0.5$ \\
\hline 21 & $4.7 \pm 0.8$ & $2.9 \pm 0.4$ & $4.1 \pm 0.7$ \\
\hline 22 & $1.1 \pm 0.3$ & $1.2 \pm 0.2$ & $1.1 \pm 0.2$ \\
\hline 24 & $2.8 \pm 0.6$ & $4.9 \pm 1.0$ & $3.2 \pm 0.6$ \\
\hline 26 & $4.8 \pm 1.2$ & $3.0 \pm 0.4$ & $4.3 \pm 0.9$ \\
\hline 27 & $3.7 \pm 0.4$ & $1.9 \pm 0.2$ & $2.9 \pm 0.1$ \\
\hline 28 & $3.8 \pm 0.8$ & $3.3 \pm 0.8$ & $3.6 \pm 0.5$ \\
\hline 29 & $4.5 \pm 0.1$ & $2.4 \pm 0.7$ & $3.8 \pm 0.5$ \\
\hline 32 & $3.5 \pm 0.7$ & $4.3 \pm 1.2$ & $3.7 \pm 0.7$ \\
\hline 34 & $0.6 \pm 0.1$ & $0.8 \pm 0.1$ & $0.7 \pm 0.0$ \\
\hline 35 & $3.3 \pm 0.4$ & $1.3 \pm 0.3$ & $2.6 \pm 0.3$ \\
\hline 37 & $3.5 \pm 0.6$ & $3.8 \pm 0.3$ & $3.6 \pm 0.4$ \\
\hline 38 & $4.7 \pm 0.5$ & $4.2 \pm 0.9$ & $4.5 \pm 0.6$ \\
\hline 39 & $2.7 \pm 0.7$ & $2.3 \pm 0.6$ & $2.5 \pm 0.3$ \\
\hline 41 & $4.8 \pm 0.3$ & $4.6 \pm 0.9$ & $4.8 \pm 0.3$ \\
\hline 42 & $0.8 \pm 0.1$ & $0.5 \pm 0.1$ & $0.6 \pm 0.1$ \\
\hline 43 & $3.1 \pm 0.6$ & $3.2 \pm 0.5$ & $3.1 \pm 0.5$ \\
\hline 44 & $5.8 \pm 1.0$ & $3.4 \pm 0.5$ & $5.0 \pm 0.4$ \\
\hline 45 & $4.4 \pm 0.3$ & $1.2 \pm 0.2$ & $3.1 \pm 0.2$ \\
\hline 46 & $3.7 \pm 0.6$ & $2.4 \pm 0.5$ & $3.2 \pm 0.4$ \\
\hline 47 & $4.8 \pm 1.3$ & $2.5 \pm 0.5$ & $4.1 \pm 1.0$ \\
\hline 48 & $3.4 \pm 0.5$ & $3.3 \pm 0.9$ & $3.3 \pm 0.5$ \\
\hline 49 & $3.8 \pm 0.5$ & $2.3 \pm 0.7$ & $3.2 \pm 0.4$ \\
\hline
\end{tabular}


Table 2. continued

\begin{tabular}{|c|c|c|c|}
\hline No. & $\gamma($ soluble $\mathrm{dAb}$ in supernatant $) /(\mathrm{ng} / \mu \mathrm{L})$ & $\gamma($ soluble $\mathrm{dAb}$ in pellet $) /(\mathrm{ng} / \mu \mathrm{L})$ & $\gamma($ total soluble $\mathrm{dAb}) /(\mathrm{ng} / \mu \mathrm{L})$ \\
\hline 50 & $4.2 \pm 0.7$ & $3.8 \pm 1.1$ & $4.1 \pm 0.1$ \\
\hline 53 & $4.3 \pm 0.9$ & $3.1 \pm 0.6$ & $3.9 \pm 0.3$ \\
\hline 54 & $4.2 \pm 0.9$ & $1.8 \pm 0.4$ & $3.5 \pm 0.8$ \\
\hline 56 & $3.7 \pm 0.8$ & $2.0 \pm 0.7$ & $3.1 \pm 0.8$ \\
\hline 57 & $3.1 \pm 0.3$ & $2.4 \pm 0.3$ & $2.8 \pm 0.2$ \\
\hline 58 & $4.1 \pm 0.8$ & $2.5 \pm 0.7$ & $3.6 \pm 0.5$ \\
\hline 59 & $3.4 \pm 0.6$ & $1.8 \pm 0.5$ & $2.7 \pm 0.4$ \\
\hline 60 & $4.5 \pm 0.5$ & $3.9 \pm 1.3$ & $4.3 \pm 0.3$ \\
\hline 62 & $2.1 \pm 0.3$ & $1.4 \pm 0.1$ & $1.8 \pm 0.1$ \\
\hline 63 & $4.5 \pm 0.7$ & $3.2 \pm 0.4$ & $4.1 \pm 0.6$ \\
\hline 66 & $2.9 \pm 0.2$ & $2.7 \pm 0.4$ & $2.8 \pm 0.2$ \\
\hline 67 & $4.8 \pm 0.9$ & $2.8 \pm 0.5$ & $4.1 \pm 0.8$ \\
\hline 68 & $5.0 \pm 0.7$ & $3.1 \pm 0.6$ & $4.4 \pm 0.3$ \\
\hline 70 & $0.9 \pm 0.1$ & $1.3 \pm 0.2$ & $1.1 \pm 0.1$ \\
\hline 71 & $4.5 \pm 0.1$ & $3.8 \pm 0.6$ & $4.3 \pm 0.2$ \\
\hline 72 & $2.7 \pm 0.4$ & $1.5 \pm 0.2$ & $2.4 \pm 0.2$ \\
\hline 73 & $1.0 \pm 0.2$ & $1.2 \pm 0.0$ & $1.1 \pm 0.1$ \\
\hline 74 & $3.6 \pm 0.5$ & $2.0 \pm 0.4$ & $3.1 \pm 0.4$ \\
\hline 78 & $5.9 \pm 0.9$ & $4.0 \pm 0.2$ & $5.4 \pm 0.6$ \\
\hline 80 & $5.5 \pm 0.9$ & $3.2 \pm 0.5$ & $4.8 \pm 0.8$ \\
\hline 81 & $1.4 \pm 0.3$ & $1.3 \pm 0.3$ & $1.4 \pm 0.3$ \\
\hline 82 & $3.6 \pm 0.6$ & $4.0 \pm 0.1$ & $3.7 \pm 0.4$ \\
\hline 83 & $4.5 \pm 0.9$ & $3.3 \pm 0.7$ & $4.2 \pm 0.7$ \\
\hline 85 & $3.9 \pm 0.9$ & $3.6 \pm 0.5$ & $3.8 \pm 0.8$ \\
\hline 87 & $2.9 \pm 0.4$ & $2.6 \pm 0.7$ & $2.8 \pm 0.4$ \\
\hline 90 & $3.0 \pm 0.3$ & $2.9 \pm 0.2$ & $3.0 \pm 0.2$ \\
\hline 91 & $4.2 \pm 0.9$ & $2.2 \pm 0.2$ & $3.4 \pm 0.5$ \\
\hline 93 & $5.0 \pm 0.6$ & $3.1 \pm 0.8$ & $4.4 \pm 0.3$ \\
\hline 94 & $3.6 \pm 0.7$ & $2.4 \pm 0.3$ & $3.1 \pm 0.5$ \\
\hline \multicolumn{4}{|c|}{ Soluble expression of $\mathrm{dAb}$ in validation strains } \\
\hline 98 & $1.5 \pm 0.2$ & $3.3 \pm 0.3$ & $2.5 \pm 0.3$ \\
\hline 99 & $3.1 \pm 0.2$ & $3.5 \pm 0.3$ & $3.3 \pm 0.2$ \\
\hline 101 & $3.1 \pm 0.2$ & $3.3 \pm 0.5$ & $3.2 \pm 0.1$ \\
\hline 102 & $3.2 \pm 0.4$ & $3.5 \pm 0.3$ & $3.4 \pm 0.3$ \\
\hline 104 & $3.8 \pm 0.5$ & $2.9 \pm 0.5$ & $3.4 \pm 0.0$ \\
\hline 105 & $3.1 \pm 0.2$ & $4.0 \pm 0.8$ & $3.5 \pm 0.4$ \\
\hline 107 & $2.7 \pm 0.3$ & $2.9 \pm 0.7$ & $2.8 \pm 0.1$ \\
\hline 108 & $3.0 \pm 0.2$ & $3.0 \pm 0.3$ & $2.8 \pm 0.1$ \\
\hline 109 & $1.1 \pm 0.3$ & $3.2 \pm 0.3$ & $2.2 \pm 0.1$ \\
\hline 110 & $3.7 \pm 0.5$ & $2.7 \pm 0.5$ & $3.2 \pm 0.3$ \\
\hline
\end{tabular}

Results are expressed as mean value \pm standard deviation

Table 3. Category of amino acids based on Vector NTI ${ }^{\circledR}$ (25)

\begin{tabular}{lc} 
Category & Amino acid \\
Charged & $R, K, D, C, H, Y, E$ \\
Polar & $\mathrm{N}, \mathrm{T}, \mathrm{C}, \mathrm{G}, \mathrm{Q}, \mathrm{S}, \mathrm{Y}$ \\
Hydrophobic & $\mathrm{A}, \mathrm{V}, \mathrm{L}, \mathrm{I}, \mathrm{F}, \mathrm{W}$ \\
Acidic & $\mathrm{D}, \mathrm{E}$ \\
Basic & $\mathrm{K}, \mathrm{R}$ \\
\hline
\end{tabular}

\section{RESULTS}

AA composition significantly affects the soluble expression of $d A b s$

It is widely accepted that AA sequence is significantly correlated with protein production, which was also shown in this study through analysis of the consistency of cluster results based on $A A$ sequences and the corresponding soluble expression levels of dAbs (Table 4). AA compositions of the whole $\mathrm{dAb}$ (in percentage) were set as variables to explore 
Table 4. Clustering results based on amino acid sequences and soluble expression amounts

\begin{tabular}{|c|c|c|c|c|c|c|c|c|c|c|c|}
\hline No. & $\begin{array}{c}\text { Expresion } \\
\text { level }\end{array}$ & Seq. & Consist. & No. & $\begin{array}{c}\text { Expresion } \\
\text { level }\end{array}$ & Seq. & Consist. & No. & $\begin{array}{c}\text { Expresion } \\
\text { level }\end{array}$ & Seq. & Consist. \\
\hline 1 & 1 & 1 & + & 34 & 2 & 2 & + & 62 & 2 & 1 & - \\
\hline 2 & 1 & 1 & + & 35 & 2 & 2 & + & 63 & 1 & 1 & + \\
\hline 3 & 1 & 1 & + & 37 & 1 & 1 & + & 66 & 2 & 2 & + \\
\hline 4 & 1 & 1 & + & 38 & 1 & 1 & + & 67 & 1 & 1 & + \\
\hline 5 & 1 & 1 & + & 39 & 2 & 1 & - & 68 & 1 & 1 & + \\
\hline 8 & 2 & 2 & + & 41 & 1 & 2 & - & 70 & 2 & 2 & + \\
\hline 9 & 1 & 1 & + & 42 & 2 & 2 & + & 71 & 1 & 1 & + \\
\hline 10 & 1 & 1 & + & 43 & 1 & 1 & + & 72 & 2 & 2 & + \\
\hline 11 & 1 & 2 & - & 44 & 1 & 1 & + & 73 & 2 & 2 & + \\
\hline 13 & 1 & 1 & + & 45 & 2 & 1 & - & 74 & 2 & 2 & + \\
\hline 15 & 2 & 1 & - & 46 & 2 & 2 & + & 78 & 1 & 1 & + \\
\hline 17 & 1 & 2 & - & 47 & 1 & 2 & - & 80 & 1 & 1 & + \\
\hline 19 & 1 & 1 & + & 48 & 1 & 2 & - & 81 & 2 & 1 & - \\
\hline 20 & 1 & 1 & + & 49 & 2 & 2 & + & 82 & 1 & 1 & + \\
\hline 21 & 1 & 1 & + & 50 & 1 & 1 & + & 83 & 1 & 1 & + \\
\hline 22 & 2 & 2 & + & 53 & 1 & 1 & + & 85 & 1 & 1 & + \\
\hline 24 & 1 & 1 & + & 54 & 2 & 1 & - & 87 & 2 & 1 & - \\
\hline 26 & 1 & 1 & + & 56 & 2 & 1 & - & 90 & 1 & 1 & + \\
\hline 27 & 2 & 2 & + & 57 & 2 & 2 & + & 91 & 1 & 2 & - \\
\hline 28 & 1 & 2 & - & 58 & 1 & 2 & - & 93 & 1 & 1 & + \\
\hline 29 & 1 & 2 & - & 59 & 2 & 2 & + & 94 & 2 & 1 & - \\
\hline 32 & 1 & 2 & - & 60 & 1 & 1 & + & Ori & 2 & 1 & - \\
\hline
\end{tabular}

1 and $2=$ cluster result of groups 1 and 2 respectively, based on expression levels or sequences of domain antibodies, + and $-=$ consistency and inconsistency of these two cluster results respectively

their effect on the dAb soluble expression level by a stepwise regression analysis, and the results are summarized in Table 5.

Stepwise regression was taken to analyse AA effect on $\mathrm{dAb}$ soluble expression level in broth supernatant, in pellet lysate supernatant and total soluble $d A b$. Results showed that the combination of AAs S, R, N, D, Q, Y, F and G had a significant influence on $\mathrm{dAb}$ soluble yield in broth supernatant, with the $p$-value of 0.002 . Specifically, S, N, D and $Q$ had positive effects, with p-values of $0.0006,0.02,0.03$ and 0.05 , respectively, which means that the soluble yield of $d A b$ in broth supernatant increased with increasing content of these AAs. However, $R$ had a negative effect $(p=0.001)$, thus $d A b$ would be more difficult to express in soluble form in broth supernatant with a higher content of R. Moreover, the combined composition of $G, R, C, N, S, Y, K$ and $A$ had a significant effect on $d A b$ soluble yield in the pellet lysate ( $p=0.002)$. Again, $R$ showed a significantly negative effect on the soluble expression $(p=0.02)$, while G, C, N and S showed significantly positive effects, with $p$-values of $0.01,0.02,0.03$ and 0.03 , respectively. When analyzing $A A$ effect on total amount of soluble $d A b$, the combined composition of $R, S, G, N, Y, C, Q$ and $F$ showed a significant influence $(p=0.0007)$. The most significant AAs were $R$ (negative), $\mathrm{S}$ (positive) and $\mathrm{G}$ (positive), for which the $\mathrm{p}$-values were $0.0008,0.006$ and 0.03 , respectively (Table 2 ). Additionally, stepwise regression analysis of the features of the dAbs, including charge, polarity, hydrophobicity, acidity and alkalinity,

Table 5. Amino acids (AAs) that have significantly effect on soluble expression levels of human serum albumin (HSA) domain antibody (dAb)

\begin{tabular}{|c|c|c|c|c|c|c|c|c|}
\hline Location & $A A$ & Effect & Location & $A A$ & Effect & Location & $A A$ & Effect \\
\hline \multirow{8}{*}{ Supernatant } & G & + & \multirow{8}{*}{ Pellet } & $\mathrm{S}$ & + & \multirow{8}{*}{ Total } & $\mathrm{R}$ & - \\
\hline & $\mathrm{R}$ & - & & $\mathrm{R}$ & - & & $\mathrm{S}$ & + \\
\hline & C & + & & $\mathrm{N}$ & + & & G & + \\
\hline & $\mathrm{N}$ & + & & D & + & & $\mathrm{N}$ & / \\
\hline & $\mathrm{S}$ & + & & Q & + & & $Y$ & / \\
\hline & $\mathrm{Y}$ & / & & $\mathrm{Y}$ & / & & $\mathrm{C}$ & / \\
\hline & $\mathrm{K}$ & / & & $\mathrm{F}$ & / & & Q & / \\
\hline & $A$ & / & & G & / & & $\mathrm{F}$ & / \\
\hline
\end{tabular}

$+=$ positively/negatively correlated,,$=$ negatively correlated, /=no correlation 
showed that polarity was the most important feature that had a positive influence on $d A b$ soluble yield $(p=0.02)$.

A linear model was built using total soluble $d A b$ yield data:

$$
\begin{aligned}
& y=-0.5810 \cdot R-0.3711 \cdot F+0.4278 \cdot G+0.4233 \cdot S+ \\
& +0.2783 \cdot C+0.2737 \cdot Q+0.2519 \cdot Y+0.2267 \cdot N
\end{aligned}
$$

where $y$ indicates the soluble expression score in $\%, \mathrm{R}$ is arginine, $\mathrm{F}$ is phenylalanine, $\mathrm{G}$ is glicine, $\mathrm{S}$ is serine, $\mathrm{C}$ is cysteine, $\mathrm{Q}$ is glutamine, $\mathrm{Y}$ is tyrosine and $\mathrm{N}$ is asparagine.

The higher the score, the higher the soluble expression level of $\mathrm{dAb}$. Clustering results divided the sequences of the 65 experimental subjects and the control dAb into high- and low-expression groups; the score distribution is shown in Fig. 2. Twenty out of $25 \mathrm{dAbs}$ belonging to the low-expression group had a score $<2.5$, while 31 out of 41 high-expression $\mathrm{dAbs}$ had a score $>2.5$. We conclude that dAbs with a score $<2.5$ are likely to be expressed at a low level in soluble form and the soluble yield would possibly be $<(2.4 \pm 0.9) \mu \mathrm{g} / \mathrm{g}$. On the other hand, dAbs with a score $>2.5$ are likely to be expressed at a high level in soluble form, with the potential soluble yield higher than $(4.0 \pm 0.5) \mu \mathrm{g} / \mathrm{g}$.

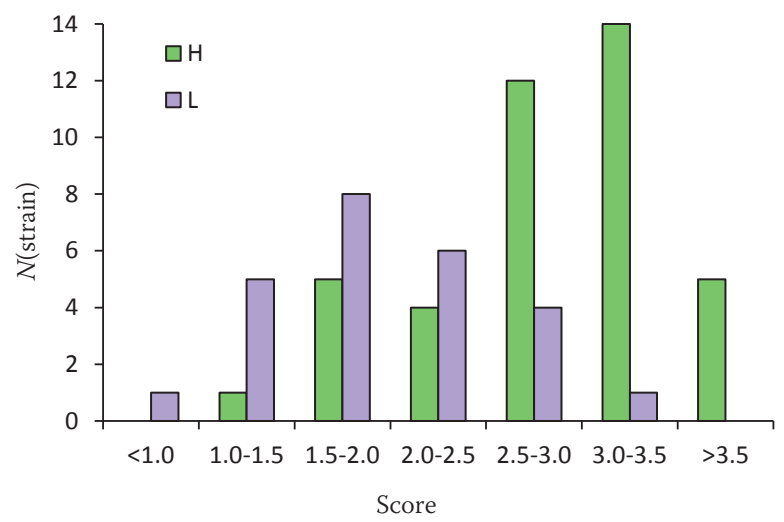

Fig. 2. Score statistics of 66 domain antibodies (dAbs). Twenty out of $25 \mathrm{dAbs}$ belonging to the low-expression group had a score $<2.5$, while 31 out of 41 high-expression dAbs had a score $>2.5$

\section{Verification}

Using the same cultivation and detection methods as in the experiments above, expression data were obtained for 10 verification subjects and a control. Comparing the predicted expression levels from the model with the actual soluble yield of these dAbs, the accuracy of the prediction model was 80 $\%$ (Table 6).

\section{DISCUSSION}

Since 1990 there have been many researches exploring the correlation between protein sequence and expression level; however, no consensus has been reached. For example, one project studied 81 different human proteins and came to the conclusion that increasing the average charge, decreasing the number of turn-forming AAs, or decreasing the content of cysteine could reduce the amount of inclusion bodies (10), while another studied G-protein-coupled receptors and found that increasing the positive charge encouraged the formation of inclusion bodies (11). Goh et al. (28) discovered that high hydrophobicity was a disadvantage for expressing proteins in soluble form by analyzing 27267 proteins selected from TargetDB, whereas Luan et al. (29) expressed 10167 ORFs of Caenorhabditis elegans using a robotic pipeline and found that hydrophobicity was not linearly correlated with the soluble expression level of protein, but proteins with lower hydrophobicity displayed higher levels of soluble expression. These works proved that studies using different subjects could come to different or even opposite conclusions. Here, to avoid the influence of protein properties including molecular mass, length and complex structures, expression system used, or operation bias, first we used dAb as the experimental subject, because this protein has low molecular mass, concentrated regions of variation, is easy to express in E. coli and has a simple tertiary structure. Second, 15 AA mutated in CDRs guaranteed enough variation among $\mathrm{dAbs}$ and little variation in pl, molecular mass and length, which helped us to focus on the variable of AA composition. Furthermore, we used consistent cultivation conditions and detection methodology to collect data, and repeated the process three times with constant control strain, which guaranteed the parallelity of operation.

\begin{tabular}{|c|c|c|c|c|c|}
\hline \multirow{2}{*}{ No. } & \multirow{2}{*}{ Score } & \multirow{2}{*}{ Prediction level } & \multicolumn{2}{|c|}{ Yield } & \multirow{2}{*}{ Consistency } \\
\hline & & & $w /(\mu \mathrm{g} / \mathrm{g})$ & Level & \\
\hline V1 & 0.5 & Low & 2.5 & Low & Yes \\
\hline V3 & 3.1 & High & 3.2 & Low & No \\
\hline V4 & 3.9 & High & 3.4 & High & Yes \\
\hline V5 & 2.6 & High & 3.4 & High & Yes \\
\hline V6 & 2.2 & Low & 3.4 & High & No \\
\hline V7 & 2.1 & Low & 2.8 & Low & Yes \\
\hline V8 & 1.1 & Low & 2.8 & Low & Yes \\
\hline V9 & 1.5 & Low & 2.2 & Low & Yes \\
\hline V10 & 1.3 & Low & 3.2 & Low & Yes \\
\hline
\end{tabular}

Table 6. Comparison between predicted and factual soluble yield of of human serum albumin (HSA) domain antibody (dAb) 
We found that polarity had a significantly positive influence on dAb soluble yield. In other words, the total content of $\mathrm{N}, \mathrm{S}, \mathrm{C}, \mathrm{G}, \mathrm{T}, \mathrm{Q}$ and $\mathrm{Y}$ positively correlated with dAb soluble yield. This may be because in this small protein there is a high likelihood of exposure to solvent of polar AAs after folding, which enhances the solubility of the protein through proteinsolvent interaction, thus indirectly increasing the soluble expression level of the protein (30).

We discovered that arginine content had a significantly negative correlation with $\mathrm{dAb}$ soluble yield, consistent with a report that positively charged AAs could hinder the process of translation, thus bringing down the expression level (7). Stepwise regression analysis showed that the glycine content was positively correlated with dAb soluble yield, which may be attributable to the small molecular mass and polarity of $\mathrm{G}$. The significantly positive influence of $S$ supports the conclusion that polar AAs benefit dAb soluble expression. We suggest that increasing the total content of $\mathrm{G}$ and $\mathrm{S}$, or decreasing the content of $\mathrm{R}$ is helpful to improve the soluble expression level of $d A b$. Findings from this study may act as a general guide for site-directed mutagenesis of HSA dAbs or other similar dAbs/ Abs to improve the soluble expression levels, which benefits further studies such as interaction mechanism and structure research. Furthermore, considering the attractive advantages of $E$. coli as a protein expression host, our preliminary observations pave the way towards establishing more efficient $E$. coli expression strategies for desired proteins.

\section{CONCLUSION}

Certain amino acids (AAs) significantly affected the soluble expression level of domain antibody $(\mathrm{dAb})$ in the broth supernatant and in the pellet lysate, and total soluble $d A b$, with the specific $A A$ combinations being $(S, R, N, D, Q),(G, R$, $C, N, S)$ and $(R, S, G)$. $R$ displayed a negative influence, whereas $\mathrm{G}$ and $\mathrm{S}$ showed positive effects. Increasing the content of polar AAs, especially $G$ and S, and decreasing the content of $\mathrm{R}$ was helpful to improve the soluble expression level of human serum albumin (HSA) dAb. This linear model had a prediction accuracy of $80 \%$.

\section{ACKNOWLEDGEMENTS}

We thank all the co-workers who assisted in the screening of mutated sequences, which is indeed a labour-intensive work.

This study was supported by the National Natural Science Foundation of China (grant nos. 31570034 and 31471251), Natural Science Foundation of Jiangsu Province (grant nos. BK20150148 and BK20161130), the Six Talent Peaks Project in Jiangsu Province (grant no. SWYY-128), National Science and Technology Major project (no. 2018ZX10302205-004-002), the 111 Project (no. 111-2-06), the Collaborative Innovation Center of Jiangsu Modern Industrial Fermentation, and the Priority Academic Program Development of Jiangsu Higher Education Institutions.

\section{REFERENCES}

1. Gräslund S, Nordlund P, Weigelt J, Hallberg BM, Bray J, Gileadi $\mathrm{O}$, et al. Protein production and purification. Nat Methods. 2008;5(2):135-46.

https://doi.org/10.1038/nmeth.f.202

2. Trevino SR, Scholtz JM, Pace CN. Amino acid contribution to protein solubility: Asp, Glu, and Ser contribute more favorably than the other hydrophilic amino acids in RNase Sa. J Mol Biol. 2007;366(2):449-60. https://doi.org/10.1016/j.jmb.2006.10.026

3. Sonoda H, Kumada Y, Katsuda T, Yamaji H. Effects of cytoplasmic and periplasmic chaperones on secretory production of single-chain Fv antibody in Escherichia coli. J Biosci Bioeng. 2011;111(4):465-70.

https://doi.org/10.1016/j.jbiosc.2010.12.015

4. Sun W, Xie J, Lin H, Mi S, Li Z, Hua F, Hu Z. A combined strategy improves the solubility of aggregation-prone single-chain variable fragment antibodies. Protein Expr Purif. 2012;83(1):21-9.

https://doi.org/10.1016/j.pep.2012.02.006

5. Studier FW. Protein production by auto-induction in high-density shaking cultures. Protein Expr Purif. 2005;41(1):20734.

https://doi.org/10.1016/j.pep.2005.01.016

6. Chang CCH, Song J, Tey BT, Ramanan RN. Bioinformatics approaches for improved recombinant protein production in Escherichia coli: Protein solubility prediction. Brief Bioinform. 2014;15(6):953-62. https://doi.org/10.1093/bib/bbt057

7. Price WN, Handelman SK, Everett JK, Tong SN, Bracic A, Luff JD, et al. Large-scale experimental studies show unexpected amino acid effects on protein expression and solubility in vivo in E. coli. Microb Inform Exp. 2011;1:6. https://doi.org/10.1186/2042-5783-1-6

8. Tian Y, Deutsch C, Krishnamoorthy B. Scoring function to predict solubility mutagenesis. Algorithms Mol Biol. 2010;5:33. https://doi.org/10.1186/1748-7188-5-33

9. Habibi N, Mohd Hashim SZ, Norouzi A, Samian MR. A review of machine learning methods to predict the solubility of overexpressed recombinant proteins in Escherichia coli. BMC Bioinformatics. 2014;15:134.

https://doi.org/10.1186/1471-2105-15-134

10. Harrison RG, Bagajewicz MJ. Predicting the solubility of recombinant proteins in Escherichia coli. In: García-Fruitós $\mathrm{E}_{\text {, }}$ editor. Insoluble proteins, methods in molecular biology (Methods and protocols), vol. 1258. New York, NY, USA: Humana Press; 2015. pp. 403-8.

https://doi.org/10.1007/978-1-4939-2205-5_23

11. Kiefer H, Vogel R, Maier K. Bacterial expression of G-protein-coupled receptors: Prediction of expression levels from sequence. Receptors Channels. 2000;7(2):109-19.

12. Idicula-Thomas $S$, Balaji PV. Understanding the relationship between the primary structure of proteins and its propensi- 
ty to be soluble on overexpression in Escherichia coli. Protein Sci. 2005; 14(3):582-92.

https://doi.org/10.1110/ps.041009005

13. Chan WC, Liang PH, Shih YP, Yang UC, Lin WC, Hsu CN. Learning to predict expression efficacy of vectors in recombinant protein production. BMC Bioinformatics. 2010;11(Suppl 1):S21.

https://doi.org/10.1186/1471-2105-11-S1-S21

14. Idicula-Thomas S, Kulkarni AJ, Kulkarni BD, Jayaraman VK, Balaji PV. A support vector machine-based method for predicting the propensity of a protein to be soluble or to form inclusion body on overexpression in Escherichia coli. Bioinformatics. 2006;22(3):278-84.

https://doi.org/10.1093/bioinformatics/bti810

15. Smialowski P, Martin-Galiano AJ, Mikolajka A, Girschick T, Holak TA, Frishman D. Protein solubility: Sequence based prediction and experimental verification. Bioinformatics. 2007;23(19):2536-42.

https://doi.org/10.1093/bioinformatics/btl623

16. Magnan CN, Randall A, Baldi P. SOLpro: Accurate sequence-based prediction of protein solubility. Bioinformatics. 2009;25(17):2200-7.

https://doi.org/10.1093/bioinformatics/btp386

17. Agostini F, Cirillo D, Livi CM, Delli Ponti R, Tartaglia GG. cc SOL omics: A webserver for solubility prediction of endogenous and heterologous expression in Escherichia coli. Bioinformatics. 2014;30(20):2975-7.

https://doi.org/10.1093/bioinformatics/btu420

18. Smialowski P, Doose G, Torkler P, Kaufmann S, Frishman D. PROSO II - A new method for protein solubility prediction. FEBS J. 2012;279(12):2192-200.

https://doi.org/10.1111/j.1742-4658.2012.08603.x

19. Holt LJ, Herring C, Jespers LS, Woolven BP, Tomlinson IM. Domain antibodies: Proteins for therapy. Trends Biotechnol. 2003;21(11):484-90. https://doi.org/10.1016/j.tibtech.2003.08.007

20. SWISS-MODEL, Protein Structure Bioinformatics Group, Swiss Institute of Bioinformatics, Biozentrum, University of
Basel, Switzerland; 2017. Available from: https://swissmodel.expasy.org/.

21. PyMOL Molecular Graphics System, v. 0.99rc6, Schrödinger, LLC, Cambridge, MA, USA. Available from: https://pymol.org/.

22. Weisser NE Hall JC. Applications of single-chain variable fragment antibodies in therapeutics and diagnostics. Biotechnol Adv. 2009;27(4):502-20.

https://doi.org/10.1016/j.biotechadv.2009.04.004

23. Holt LJ, Basran A, Jones K, Chorlton J, Jespers LS, Brewis ND, Tomlinson IM. Anti-serum albumin domain antibodies for extending the half-lives of short lived drugs. Protein Eng Des Sel. 2008;21(5):283-8.

https://doi.org/10.1093/protein/gzm067

24. R software, v. 3.3.2, Auckland, New Zealand: Statistics Department of the University of Auckland; 2017. Available from: https://www.r-project.org/.

25. Vector $\mathrm{NTI}^{\circledR}{ }^{\circledR}$, v. 11.5, Thermo Fisher Scientific, Waltham, MA, USA; 2011. Available from: https://www.thermofisher.com/ cn/zh/home/life-science/cloning/vector-nti-software.html.

26. Clustal Omega, Cambridgeshire, UK: EMBL-EBI, European Molecular Biology Laboratory; 2017. Available from: https:// www.ebi.ac.uk/Tools/msa/clustalo/.

27. Aho K, Derryberry D, Peterson T. Model selection for ecologists: The worldviews of AIC and BIC. Ecology. 2014;95(3):631-6.

https://doi.org/10.1890/13-1452.1

28. Goh CS, Lan N, Douglas SM, Wu B, Echols N, Smith A, et al. Mining the structural genomics pipeline: Identification of protein properties that affect high-throughput experimental analysis. J Mol Biol. 2004;336(1):115-30. https://doi.org/10.1016/j.jmb.2003.11.053

29. Luan CH, Qiu SH, Finley JB, Carson M, Gray RJ, Huang W, et al. High-throughput expression of $C$. elegans proteins. Genome Res. 2004;14:2102-10. https://doi.org/10.1101/gr.2520504

30. Lesser GJ, Rose GD. Hydrophobicity of amino acid subgroups in proteins. Proteins. 1990;8(1):6-13. https://doi.org/10.1002/prot.340080104 